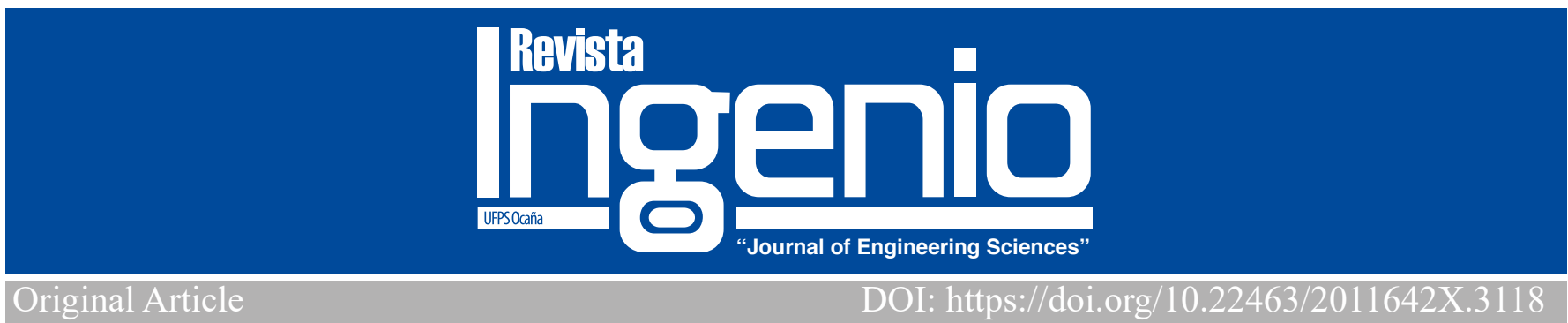

\title{
Estado de vórtices en un cuadrado superconductor de dos-orbitales con condiciones de contorno mixtas
}

\author{
Vortex state in a superconducting two-orbital square with mixed boundary conditions
}

Ph.D Cristian Andrés Aguirre Tellez', Ph.D Ely Dannier Valbuena Niño², Ph.D José José Barba Ortega ${ }^{3}$

${ }^{I}$ Grupo de Fisica Mesoscópica, Universidade Federal do Rio Grande do Sul, Porto Alegre, Brasil, Orcid: https://orcid.org/0000-0001-8064-6351, Email: cristian@fisica.ufmt.br

${ }^{2}$ Materials Science and Technology Research Group, Foundation of Researches in Science and Technology of Materials, Bucaramanga, Colombia, Orcid: https://orcid.org/0000-0003-4154-7179, Email: edvnino@foristom.org

${ }^{3}$ Grupo de Física Mesoscópica, Departamento de Física, Universidad Nacional de Colombia, Bogotá, Colombia, Orcid: https://orcid.org/0000-0003-3415-1811, Email: jjbarbao@unal.edu.co

Como citar: C.A. Aguirre-Tellez, E.D. Valbuena-Niño \& J.J. Barba-Ortega, "Estado de vórtices en un cuadrado superconductor de dos-orbitales con condiciones de contorno mixtas", Revista Ingenio, vol 15 (1), pp.38-43, 2018

Fecha de recibido: 15 de mayo de 2017 Fecha aprobación: 27 de octubre de 2017

\section{RESUMEN}

Palabras claves:

En esta contribución estudiamos el estado de vórtices en una muestra cuadrada superconductora de dos orbitales en presencia de un campo magnético externo $\mathbf{H}$ constante en un proceso de zero-field-cooling. La muestra está en contacto con diferentes tipos de

deGennes, Ginzburg-

Landau, Mesoscópico, materiales simulados a través del parámetro de extrapolación de deGennes (b) . Nuestra investigación se lleva a cabo resolviendo las ecuaciones Ginzburg-Landau dependientes del tiempo en dos dimensiones. Estudiamos la inducción magnética, densidad de electrones superconductores y la fase del parámetro de orden para diferentes valores de $b$ sobre la superficie de la muestra. Para ello, usamos dos combinaciones de condiciones de contorno para las cuatro superficies del cuadrado. Mostramos novedosas e interesante configuración de vórtices altamente dependiente de los valores escogidos de $b$, temperatura, y tamaño de la muestra.

\begin{tabular}{ll}
\hline ABSTRACT \\
\hline Key words: & $\begin{array}{l}\text { In this contribution we studied the vortex state in a two-orbital superconducting square in the presence of a constant external magnetic } \\
\text { field } \mathbf{H} \text { in a zero-field-cooling process. The sample is in contact with different types of materials simulated via deGennes extrapolation } \\
\text { parameter }(b) \text {. Our research was carried out by solving the bidimensional time-dependent Ginzburg-Landau. We study the magnetic } \\
\text { induction, superconducting electron density and the phase of the order parameter for different values of } b \text { on the sample surface. To } \\
\text { Landau, Mesoscopic, }\end{array}$ \\
Superconductor, Vortices. & configuration highly dependent on the chosen values of $b$, temperature, and sample size.
\end{tabular}

\section{Introducción}

El estado superconductor es uno de los estados de la materia condensada más interesantes debido a su resistividad eléctrica nula y a su diamagnetismo, los materiales que presentan estas propiedades son buenos candidatos para aplicaciones tecnológicas y para minimizar pérdidas energéticas por calor [1]. Infelizmente estas propiedades solo se evidencian bajo cierta temperatura crítica, que está en el orden de los Kelvin, y depende de cada material [2-4]. Así la panacea en esta rama de la física es hallar un material que sea superconductor a altas temperaturas y por que no, a temperatura y presión ambiente.

Una de las teorías mas exitosas en describir el estado superconductor es la teoría fenomenológica de
Ginzburg-landau; con esta teoría Abrikosov demostró que existen dos tipos de superconductores: tipo I, en el que el parámetro de Ginzburg-Landau $\kappa<(\sqrt{ } 2)^{-1}$, en los cuales no existe el estado de vórtices, y tipo II, en el que $\kappa<(\sqrt{2})$ 1 , en los cuales el campo magnético ingresa a las muestras de una forma cuantizada [1-4]. Históricamente, el enfoque de Bogomolny se realizó para la física de altas energías y la comunidad superconductora no lo sabía, sólo muy recientemente se utilizó el método Bogomolny para estudiar vórtices en discos mesoscópicos y calcular la estructura de vórtices multicuantes 4 en superconductores con $\kappa=(\sqrt{2})^{-1}$.

Los vórtices existentes en los superconductores de tipo II, se conocen como los vórtices de Shubnikov-Abrikosov; este tipo de vórtice transporta un número entero de cuantos 
de flujo magnético, llamado fluxoide que es definido como $\Phi_{0}=$ hc/2e. Sin embargo, en el 2001 se descubre el bromuro de magnesio $\left(M g B_{2}\right)$ con una temperatura crítica $\mathrm{T}_{c}=39$ $\mathrm{K}$ [5] y con características físicas muy diferentes a los superconductores convencionales o de banda única; este material fue llamado superconductor de dos-bandas o multicomponente porque presenta fracciones de fluxoides. Los materiales superconductores se pueden describir mediante las teorías de Ginzburg-Landau, Bardeen-Cooper-Schrieffer, Faddeev, Lawrence-Doniach, entre otras; en estos modelos una banda puede acoplarse a las otras bandas por medio de uniones tipo Josephson, y Cooper. Por ejemplo, E. Babaev y colaboradores analizaron muestras superconductoras de dos bandas con defectos topológicos mostrando la existencia de vórtices que llevan una fracción arbitraria de fluxoide; además, presentaron un estudio microscópico del comportamiento de los parámetros de orden, descrito por el modelo estándar de Bardeen-Cooper-Schrieffer [6-11].

Experimentalmente, se han estudiado materiales superconductores multi-bandas, encontrando vórtices fraccionales en un sistema de dos capas mediante la implementación de centros de anclaje en la superficie de una de las capas [12]. T. Yanagisawa y colaboradores, mostraron que un modelo de dos-orbitales o dos-bandas describe bien la conductividad óptica en películas finas de $M g B_{2}$ [13]. Teóricamente se ha estudiado la superconductividad multibandas en varios trabajos, por ejemplo, J. Barba-Ortega y colaboradores, estudiaron el efecto de la naturaleza y tamaño de anti-puntos sobre la susceptibilidad magnética en una muestra superconductora, configuración de vórtices y magnetización, encontrando estados de vórtices novedosos en el sistema para diferentes campos magnéticos externos; además, estudiaron la influencia de la longitud de extrapolación de deGennes en el estado de vórtices en un cuadrado superconductor con un defecto central, hallaron configuraciones exóticas de vórtices debido al potencial repulsivo (atractivo) de corto (largo) rango entre los flujos magnéticos y que en el límite de la película fina [14-15]. Manucharyan y colaboradores, estudiaron muestras superconductoras con una corriente tipo Josephson encontrando la existencia de una banda dominante con este tipo de unión [16].

Por otra parte, se han estudiado tres acoplamientos principales que pueden ocurrir en superconductores multibanda o multi-orbitales, que son Josephson, bi-cuadrático y débil, donde en general el primer tipo de acoplamiento es un acoplamiento débil, lo que permite tunelizar algunos pares de Cooper entre cada una de las bandas. En general, por presentar un acople débil, las principales propiedades termodinámicas en heteroestructuras superconductores modelados usando este acoplamiento, no difieren mucho de aquellas propiedades mostradas en superconductores convencionales de banda única [3-4].

En el presente artículo, estudiamos la densidad electrónica en la banda dominante en un cuadrado superconductor de dos-orbitales en presencia de un campo magnético externo; analizamos la fase del parámetro de orden $\Delta \phi$ y la inducción magnética B para diferentes condiciones de contorno (Neumann y Dirichlet), tamaño de la muestra y temperatura en un proceso de zero-field-cooling. Este proceso básicamente consta de los siguientes pasos: situamos la muestra a campo magnético nulo; seguido, se procede a enfriar la muestra por debajo de su temperatura crítica; posteriormente, se enciende el campo magnético en un valor menor al segundo campo critico termodinámico; finalmente, se realizan las medidas termo-magnéticas que se requieran, por ejemplo, magnetización en función de la temperatura, vorticidad, energía libre de Gibbs, calor específico, susceptibilidad magnética, etc.

Este artículo está organizado de la siguiente forma: en la sección 2 describimos el formalismo teórico utilizado, en la sección 3 presentamos el análisis de resultados y en la sección 4 exponemos nuestras conclusiones.

\section{Formalismo Teórico}

El sistema mostrado en la Figura 1 será estudiado resolviendo las ecuaciones Ginzburg-Landau de dos-bandas o dos-orbitales (2B-TDGL). La interacción entre bandas es mediada mediante el término $\pi$ ij; este acople tipo Josephson es dado por $\psi_{i}^{*} \psi_{j}+\psi_{i} \psi_{j}^{*}$, con el parámetro de orden $\psi_{j}=\left|\psi_{j}\right| e^{i \phi j}$ donde $\phi_{\mathrm{j}}$ es la fase del $\mathrm{j}$-th parámetro de orden [17-20].

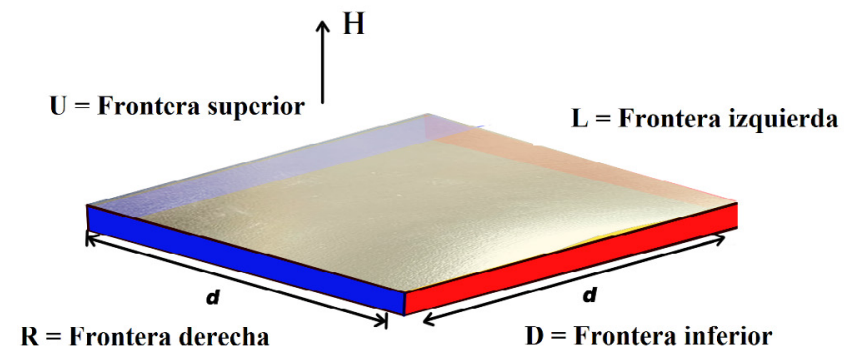

Figura 1. Esquema del sistema estudiado. Cuadrado superconductor en contavto con diferentes materiales y en presencia d eun campo magnético $\mathbf{H}$.

Las ecuaciones Ginzburg-Landau (Ecuación (1) a Ecuación (3)) fueron escaladas así: $\psi$ en unidades de $\psi_{\infty}$, longitudes en unidades de la longitud de coherencia $\xi$, el campo magnético externo en unidades del campo critico superior $H_{c 2}$, el vector potencial A en unidades de $H_{c 2} \xi$, temperatura en unidades de la temperatura critica $T_{c}$. 


$$
\begin{gathered}
\sum_{i=1}^{2} \frac{\partial \psi_{i}}{\partial t}=\sum_{i=1}^{2} H^{2} \psi_{i}+(1-T)\left(\left|\psi_{i}\right|^{2}-1\right) \psi_{i} \\
+\pi_{i j}
\end{gathered}
$$

$$
\frac{\partial \boldsymbol{A}}{\partial t}=(1-T) \sum_{i=1}^{2} \operatorname{Re}\left(\psi_{i}^{*} \boldsymbol{H} \psi_{i}\right)-k^{2} \nabla \times \nabla \times \mathbf{A}
$$

Donde:

$$
\pi_{i j}=\sum_{i=1}^{2} \sum_{i \neq j=1}^{2} 2 \mu_{i j}\left|\psi_{i}\right|\left|\psi_{j}\right| \cos \left(\phi_{i}-\phi_{j}\right)
$$

La condición de contorno general para el parámetro de orden esta definido por la Ecuación (4).

$$
\boldsymbol{n} \cdot(i \nabla+\mathbf{A}) \psi=n \cdot \boldsymbol{H} \Psi=-i \psi b^{-1}
$$

donde $\mathrm{b}$ es la longitud de extrapolación de deGennes y simula la condición de contorno [21-24], así $b \rightarrow \infty$ simula una interface superconductor-dieléctrico o superconductor vacío, para $b>0$ tenemos un superconductor en contacto con un metal $\mathrm{y} b<0$, una interface superconductor-superconductor a mayor $\mathrm{T}_{\mathrm{c}}$ se considera, todas estas situaciones son tomadas mediante la condición de contorno de Neumann; si $b=0 \psi=0$ tenemos la condición de contorno de Dirichlet lo cual simula una superficie completamente en estado normal.

Todos nuestros cálculos fueron realizados considerando un proceso zero-field-cooling a un campo magnético externo constante $\mathrm{H}=0.9$ y $\mu_{\mathrm{ij}}=0.01$. Consideramos la muestra de tamaño $\mathrm{d}=20 \xi$ y $\mathrm{d}=10 \xi$ para dos casos: Caso (a) las fronteras R y D (vea la Figura 1) están en estado completamente normal, obedeciendo la condición de contorno de Dirichlet $\mathrm{b}=0 \psi=0$, a su vez las fronteras L y U están en contacto con otro superconductor a mayor temperatura crítica simulado por $b=-2.0$, obedeciendo la condición de contorno de Neumann. Caso (b) las fronteras L y U están en contacto con un metal simulado por $\mathrm{b}=2$, y las fronteras $\mathrm{R}$ y $\mathrm{D}$ están en contacto con un dieléctrico simulado por $\mathrm{b} \rightarrow \infty$; en este caso las cuatro fronteras obedecen la condición de contorno de Neumann.

\section{Análisis de Resultados}

Estudiamos el sistema presentado en la Figura 1, para un sistema superconductor de dos-orbitales; todos nuestros resultados son mostrados para la banda dominante. Consideramos un proceso de zero-field-cooling con un campo magnético externo constante $\mathrm{H}=0.9$. En la Figura 2, presentamos el estado de vórtices o densidad electrónica $|\psi|^{2}$, donde las regiones oscuras representan valores del modulo del parámetro de orden $|\psi|^{2}=0.0$ (estado normal) y las regiones claras $|\psi|^{2}=1.0$ (estado superconductor); los cálculos fueron hechos considerando $\mathrm{T}=0.0 \mathrm{y} \mathrm{T}=0.2$.

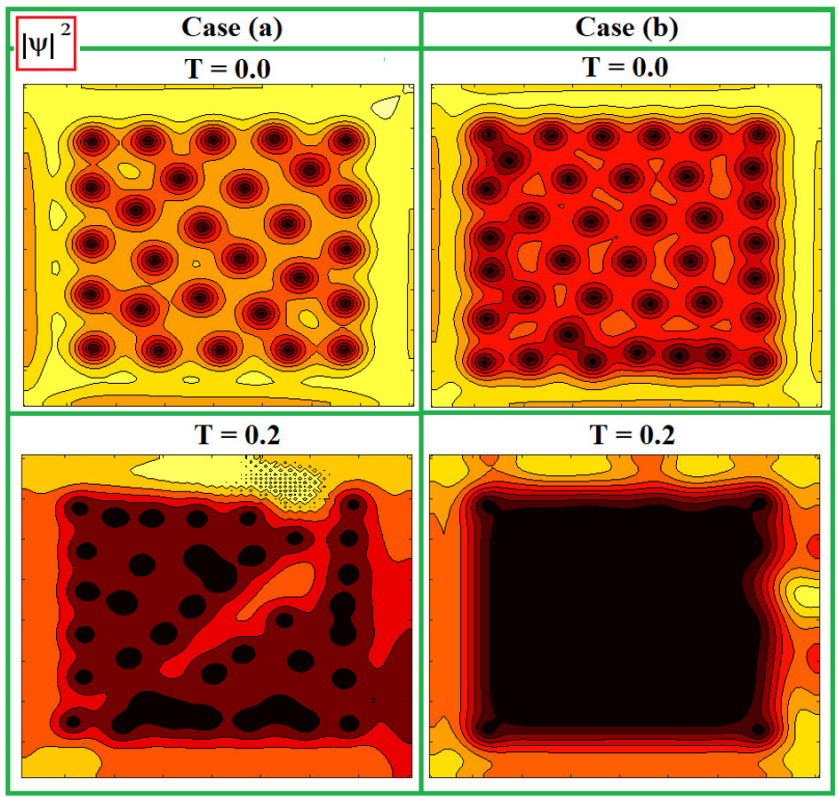

Figura 2. $|\psi|^{2}$ para los casos (a) y (b) a $\mathrm{T}=0$ (arriba) y $\mathrm{T}=0.2$ (abajo), para $\mathrm{L}=20 \xi, \kappa=1.0$. Regiones oscuras y claras representan valores del modulo del parámetro de orden de 0 a 1 .

En la Figura 3, las regiones oscuras y claras representan valores de fase $\Delta \phi$ de 0 a $2 \pi$; por lo que circulaciones a través de una trayectoria cerrada en el material identifica un cambio de fase igual $2 \pi N$, siendo $\mathrm{N}$ la vorticidad definida como $\mathrm{N}=\mathrm{n} \Phi_{0}, \mathrm{y} \mathrm{n}=1,2,3, \ldots$ Es claro ver en la Figura 2 y Figura 3 que la barrera de energía de Bean-Livingston es menor en las regiones donde la superconductividad es depreciada, es decir en $\psi=0$ o donde $\mathrm{b} \rightarrow 0_{+}$; esto conlleva a que el ingreso de los vórtices ocurra preferiblemente por las fronteras $R$ y $U$ en los dos casos estudiados, y es independiente de la temperatura y tamaño de la muestra. 


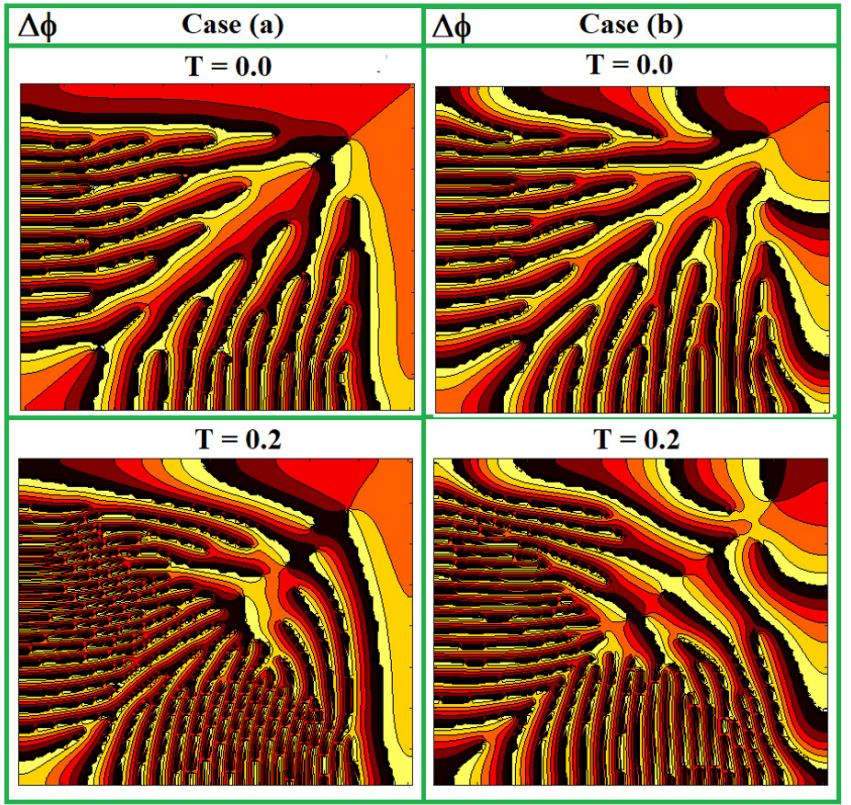

Figura 3. $\Delta \phi$ para los casos (a) y (b) a $\mathrm{T}=0$ (arriba) y $\mathrm{T}=0.2$ (abajo), para $\mathrm{L}=20 \xi, \kappa=1.0$. Regiones oscuras y claras representan valores de $\Delta \phi / 2 \pi$ de 0 a 1 .

En la Figura 4 se observa que la presencia de materiales diferentes sobre las superficies de la muestra genera una gran diferencia en la configuración de vórtices en un proceso zero-field-Cooling, donde la muestra es enfriada bajo su temperatura critica y llevada a su estado superconductor a un campo magnético constante. Es importante notar que en el núcleo del vórtice $\psi \rightarrow 0, \mathrm{~B} \rightarrow \mathrm{B}_{\max }$, $\mathrm{y}$ en el estado superconductor $\mathrm{o}$ fuera del vórtice $\psi \rightarrow \psi_{\infty} \mathrm{y} \mathrm{B} \rightarrow 0$. Las regiones claras en la Figura 4, muestran un ingreso total del campo magnético dentro de la muestra, es decir el estado superconductor ha sido completamente destruido y no existe información coherente sobre el numero de vórtices que pueden existir dentro de la muestra; para ello es necesario contar la variación de la fase en una circulación sobre todo el cuadrado.

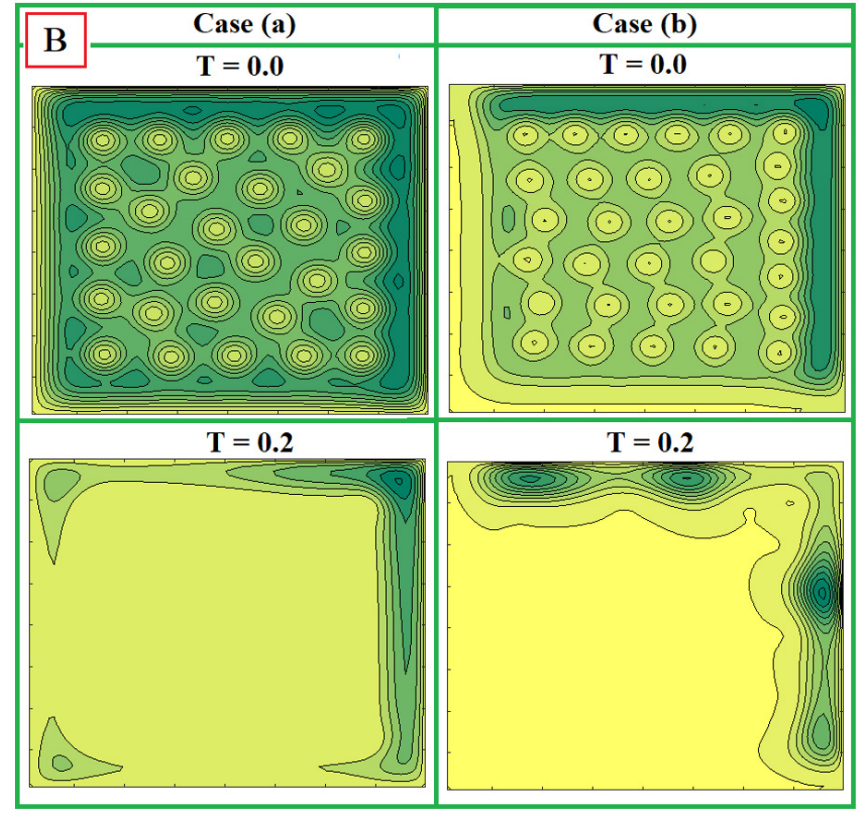

Figura 4. B para los mismos casos mostrados en las Figura $1 \mathrm{y}$ Figura 2; $\mathrm{L}=20 \xi, \mathrm{k}=1.0$.

En la Figura 5, presentamos la configuración de vórtices, fase $\Delta \phi$ e inducción magnética $\mathrm{B}$ para $\mathrm{T}=0.0, \mathrm{~L}=10 \xi, \kappa=1.0$ (correspondiente a una muestra de $\mathrm{Nb}$ con $\xi(0)=\lambda(\mathrm{T}=0)=38$ nm) y $\kappa=5.0$ (correspondiente a una aleación $\mathrm{Pb}-\mathrm{In}$ con $\lambda(\mathrm{T}=0)=100 \mathrm{~nm})$.

La Figura 5 muestra que, al disminuir el tamaño de la muestra, disminuye la vorticidad. Por consiguiente, para $\mathrm{L}=10 \xi$ se obtiene $\mathrm{N}=4$ y $\Delta \phi=8 \pi$.

Es interesante notar que cuando se escoge una interfaz con otro superconductor a mayor temperatura crítica, los efectos de proximidad de los pares de Cooper en la frontera hacen que haya menos área disponible para la formación de los vórtices. En la Figura 4 se muestra que los vórtices están mas próximos cuando se considera la condición de contorno de Dirichlet, es decir se tiene una mayor barrera de energía para $b<0$. 


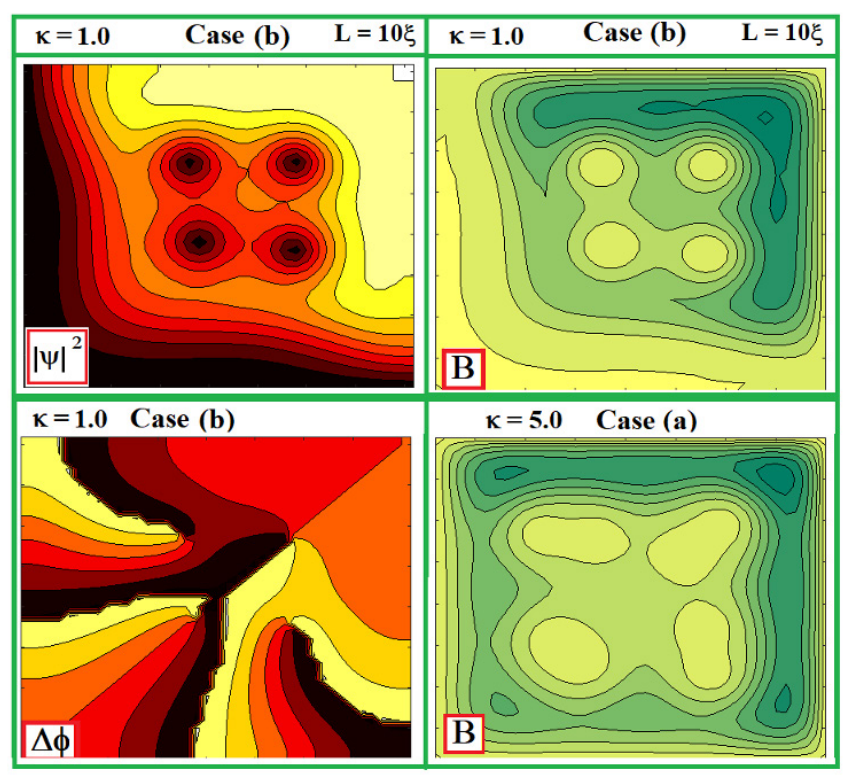

Figura 5. $|\psi|^{2}, \Delta \phi$ y $\mathrm{B}$, para $\mathrm{L}=10 \xi, \mathrm{T}=0.0$.

En la Figura 6, presentamos la configuración de vórtices, fase $\Delta \phi$ e inducción magnética $\mathrm{B}$ para $\mathrm{T}=0.0$ y $\mathrm{L}=10 \xi$, y $L=20 \xi, \kappa=5.0$; en la Figura 6 se observa que cuando el parámetro Ginzburg-Landau aumenta, la vorticidad aumenta. Para $\mathrm{L}=20 \xi$ se obtiene $\mathrm{N}=46$ y $\Delta \phi=92 \pi$; mientras que para $\mathrm{L}=10 \xi$ se obtiene $\mathrm{N}=8$ y $\Delta \phi=32 \pi$. Este hecho se evidencia al ver $\xi=\kappa \lambda$; si $\kappa$ aumenta, la longitud de coherencia $\xi$ disminuye para un valor constante de la longitud de penetración $\lambda$, y como $\xi$ es el tamaño característico de los vórtices, es natural que si aumenta $\kappa$ disminuye el tamaño del vórtice permitiendo un mayor número de ellos en el área de la muestra.

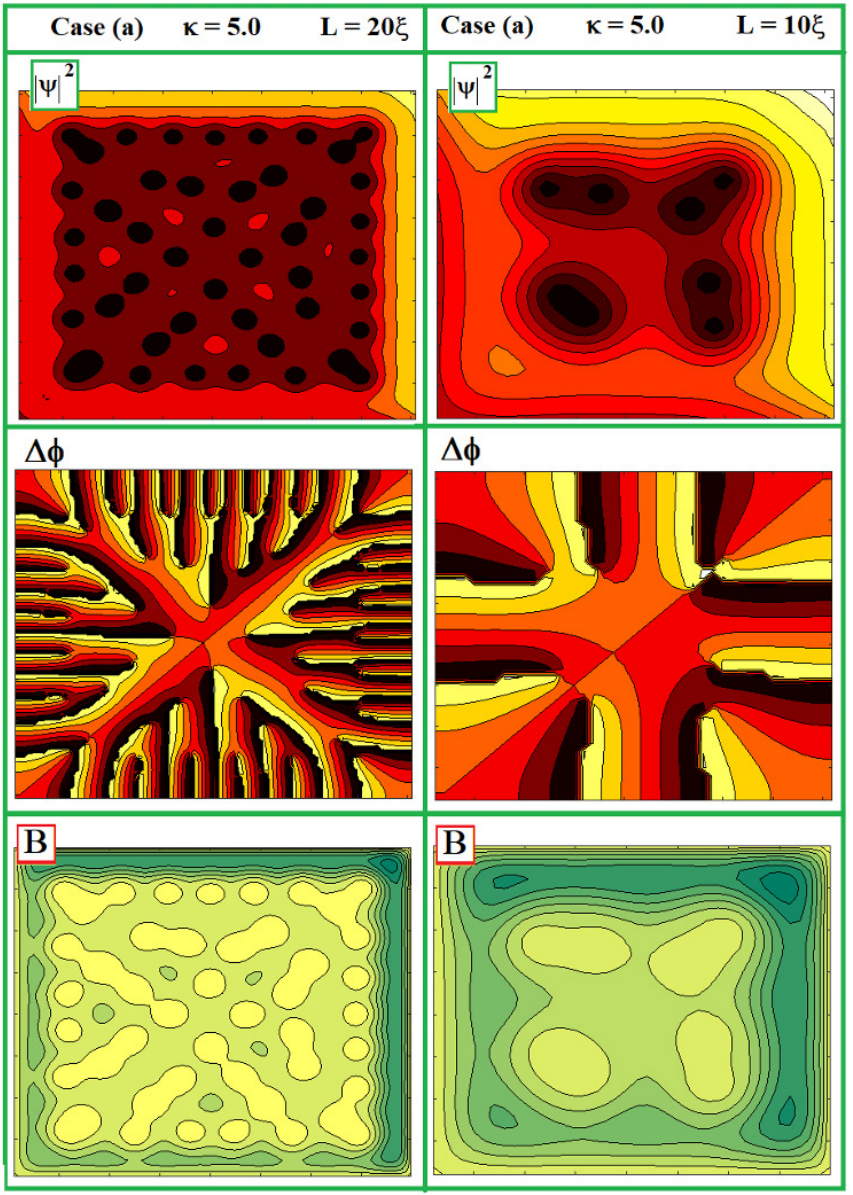

Figura 6. $|\psi|^{2}, \Delta \phi$ y $\mathrm{B}$, para $\mathrm{L}=20 \xi, \mathrm{L}=10 \xi, \kappa=5.0, \mathrm{~T}=0.0$.

\section{Conclusiones}

Solucionando el sistema de ecuaciones Giznburg-Landau para un sistema superconductor de dos-orbitales considerando acople Josephson, estudiamos el efecto de las condiciones de contorno (Neumann y Dirichlet) sobre la configuración de vórtices en un cuadrado inmerso en un campo magnético constante en un proceso de zero-field-cooling. Estudiamos el efecto de estas condiciones de contorno no homogéneas sobre el parámetro de orden, su fase y la inducción magnética; obtuvimos estados estacionarios no convencionales en la banda dominante. Encontramos que el ingreso de vórtices siempre ocurre por las regiones donde la superconductividad es depreciada, es decir aquella frontera que obedece la condición de contorno de Neuman, o donde la barrera de energía de Bean-Leavingston es menor; al aumentar el tamaño de la muestra, y el parámetro de Ginzburg-Landau un mayor numero de vórtices penetra en ella ya que la longitud de coherencia disminuye. Por consiguiente, consideramos que nuestros resultados aportan significativamente a los trabajos experimentales que busquen la manipulación de vórtices mediante la implementación de heteroesturcturas superconductoras-normal para minimizar el gasto energético y que sean aplicables en las nuevas tecnologías. 


\section{Agradecimientos}

Los autores agradecen a las diosas por mostrarnos la belleza de la naturaleza y permitimos tener una buena vida.

\section{Referencias}

[1] A. A. Abrikosov, "On the magnetic properties of superconductors of the second group", Soviet Physics, JETP, vol. 5, pp. 1174-1182, June 1957.

[2] L. Kramer, "Thermodynamic Behavior of Type-II Superconductors with Small $\kappa$ near the Lower Critical Field”, Physical Review B, vol. 3, pp. 3821-3825, June 1971.

[3] J. Barba-Ortega, E. Sardella, "Superconducting properties of a mesoscopic parallelepiped with anisotropic surface conditions", Physics Letters A, vol. 379, pp. 3130-3135, October 2015.

[4] J. Barba-Ortega, Edson Sardella, J. A. Aguiar, "Superconducting boundary conditions for mesoscopic circular samples", Superconductor Science and Technology, vol. 24, no. 015001, pp. 1-7, December 2010.

[5] J. Nagamatsu, N. Nakagawa, T. Muranaka, Y. Zenitani, J. Akimitsu, "Superconductivity at $39 \mathrm{~K}$ in magnesium diboride", Nature, vol. 410, pp. 63-64, March 2001.

[6] E. Babaev, "Vortices with Fractional Flux in Two-Gap Superconductors and in Extended Faddeev Model", Physical Review Letters, vol. 89, no. 6, pp. 06700110670014, August 2002.

[7] Q. Meng, C. N. Varney, H. Fangohr, E. Babaev, "Phase diagrams of vortex matter with multi-scale inter-vortex interactions in layered superconductors", Journal of Physics: Condensed Matter, vol. 29, no. 035602, pp. 1-16, November 2016.

[8] M. Silaev, E. Babaev, "Microscopic theory of type-1.5 superconductivity in multi-band systems", Physical Review B, vol. 84, no. 094515, pp. 1-9, September 2011.

[9] M. Silaev, E. Babaev, "Microscopic derivation of twocomponent Ginzburg-Landau model and conditions of its applicability in two-band systems", Physical Review B, vol. 85, no. 134514, pp. 1-12, April 2012.

[10] J. Carlström, E. Babaev, M. Speight, "Type-1.5 superconductivity in multiband systems: Effects of interband couplings", Physical Review B, vol. 83, no. 174509, pp. 1-17, May 2011.

[11] T. Yanagisawa, "Topological Solitons in ThreeBand Superconductors with Broken Time Reversal Symmetry", Journal of the Physical Society of Japan, vol. 79, no. 6, 063708, 1-6, June 2010.

[12] F. S. Portela, L. T. Corredor, P. Barrozo, S. G. Jung, G. Zhang, J. Vanacken, V. V. Moshchalkov, J. A. Aguiar, "Superconducting properties of $\mathrm{Nb} / \mathrm{Pb} / \mathrm{Nb}$ trilayer", Superconductor Science and Technology, vol. 28, no.
034001, pp. 1-4, January 2015.

[13] T. Yanagisawa, H. Shibata, "Orbital-Dependent TwoBand Superconductivity in MgB2", Journal of the Physical Society of Japan, vol. 72, pp. 1619-1622, April 2003.

[14] C. Aguirre, M. R. Joya, J. Barba-Ortega, "Effect of Anti-dots on the Magnetic Susceptibility in a Superconducting Long Prism", Journal of Low Temperature Physics, vol. 186, pp. 250-258, November 2016.

[15] J. Barba-Ortega, E. Sardella, J. Albino Aguiar, "Vortex-Antivortex Dynamics in a Mesoscopic Superconducting Prism with a Centered Antidot", Journal of Superconductivity and Novel Magnetism, vol. 24, pp. 97-100, September 2010.

[16] V. E. Manucharyan, N. A. Masluk, A. Kamal, J. Koch, L. I. Glazman, M. H. Devoret, "Evidence for coherent quantum phase slips across a Josephson junction array", Physical Review B, vol. 85, no. 024521, pp. 1-15, January 2012.

[17] E. Babaev, J. Carlström, M. Silaev, J. M. Speight, "Type-1.5 superconductivity in multicomponent systems", Physica C, vol. 533, pp. 20-35, Febraury 2017.

[18] J. Garaud, J. Carlström, E. Babaev, “Topological Solitons in Three-Band Superconductors with Broken Time Reversal Symmetry", Physical Review Letters, vol. 107, no. 197001, pp. 1-5, November 2011.

[19] J. Garaud, M. Silaev, and E. Babaev, "Thermoelectric Signatures of Time-Reversal Symmetry Breaking States in Multiband Superconductors", Physical Review Letters, vol. 116, no. 097002, pp. 1-7, March 2016.

[20] J. Barba-Ortega, E.D. V. Niño, E. Sardella, "Parámetro

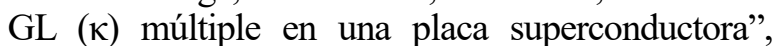
Universidad Ciencia y Tecnología, vol. 20, no. 79, pp. 87-91, June 2016.

[21] J. Barba-Ortega, E. Sardella, J. A. Aguiar, "Superconducting boundary conditions for mesoscopic circular samples", Superconductor Science and Technology, vol. 24, no. 015001, pp. 1-7, December 2010.

[22] E. Babaev, J. Carlström, M. Speight, "Type-1.5 Superconducting State from an Intrinsic Proximity Effect in Two-Band Superconductors", Physical Review Letters, vol. 105, no. 067003, pp. 1-4, August 2010.

[23] H. Fink and W. Joiner, "Surface Nucleation and Boundary Conditions in Superconductors", Physical Review Letters, vol. 23, no. 3, pp. 120-123, July 1969.

[24] V. Ginzburg, "On Surface Superconductivity", Physics Letters, vol. 13, no.2, pp. 101-102, November 1964. 